\title{
General Psychiatry Placebo effects and the molecular biological components involved
}

\author{
Lei $\mathrm{Cai},{ }^{\odot}$ Lin $\mathrm{He}$
}

To cite: Cai L, He L. Placebo effects and the molecular biological components involved. General Psychiatry 2019;32:e100089. doi:10.1136/ gpsych-2019-100089

Received 29 May 2019 Revised 18 July 2019 Accepted 30 July 2019

\section{Check for updates}

(C) Author(s) (or their employer(s)) 2019. Re-use permitted under CC BY-NC. No commercial re-use. See rights and permissions. Published by BMJ.

Bio-X Institutes, Key Laboratory for the Genetics of Developmental and Neuropsychiatric Disorders (Ministry of Education), Collaborative Innovation Center of Genetics and Development, Shanghai Jiaotong University, Shanghai 200240, China

Correspondence to Dr Lei Cai, Bio-X Institutes, Key Laboratory for the Genetics and Developmental and Neuropsychiatric Disorders (Ministry of Education), Collaborative Innovation Center of Genetics and Development, Shanghai Jiaotong University, 55 Guangyuanxi Road, Shanghai 200030, China; Icai@sjtu.edu.cn

\begin{abstract}
Pharmacologically inactive substances have been used in medicine for more than 700 years and can trigger beneficial responses in the human body, which is referred to as the placebo effects or placebo responses. This effect is robust enough to influence psychosocial and physiological responses to the placebo and to active treatments in many settings, which has led to increased interest from researchers. In this article, we summarise the history of placebo, the characteristics of placebo effects and recent advancements reported from the studies on placebo effects and highlight placebome studies to identify various molecular biological components associated with placebo effects. Although placebos have a long history, the placebome concept is still in its infancy. Although behavioural, neurobiological and genetic studies have identified that molecules in the dopamine, opioid, serotonin and endocannabinoid systems might be targets of the placebo effect, placebome studies with a no-treatment control (NTC) are necessary to identify whole-genome genetic targets. Although bioinformatics analysis has identified the molecular placebome module, placebome studies with NTCs are also required to validate the related findings.
\end{abstract}

\section{INTRODUCTION}

In the 1300s, the word 'placebo' first appeared in a Latin translation of the Hebrew Bible, with the original meaning of 'to walk'. Later, because of a mistranslation, placebo was defined as 'to please'. ${ }^{2}$ With this positive meaning, the word placebo was recorded as meaning 'to please' in early usage. In the mid-to-late $1700 \mathrm{~s}$, the term placebo began to be used as medical jargon. The prevailing opinion is that an English physician and pharmacologist, William Cullen, introduced the concept of placebo in his clinical lectures given in 1772, but another opinion is that before him, another British physician, Alexander Sutherland, who was familiar with the water cure, first used the word placebo in his book and tried to revive some ancient medical doctrines. ${ }^{34}$ At that time, according to William Cullen's introduction, the word placebo referred to drugs that were administered to satisfy a patient's desire for a remedy, however the physician thought that the placebo was ineffective for treatment of severe diseases. ${ }^{3}$

In this article, we operationally define placebo as an inert treatment, such as: drugs or surgery, which can be used to simulate administration of a real medical intervention and has been used as an indispensable control in randomised clinical trials (RCT). ${ }^{5}$ In 1784, Benjamin Franklin and Antoine Lavoisier used placebos as controls in a trial in which they exposed patients to so-called 'animal magnetism' objects or normal objects without identifying them and found that the patients' responses were similar. ${ }^{6}$ In RCTs, placebos are used as a methodological tool to challenge and debunk ineffective treatments and have become a mainstay of modern medicine. RCTs are the gold standard for tests of safety and efficacy of novel medical treatments and include definite steps, such as double-blind randomisation, besides the use of placebo. ${ }^{7}$ The ultimate goal of medical treatment is to heal (ie, to control or cure an illness) and provide symptom relief or comfort. ${ }^{8}$ However, at some point in the progression of diseases, there are no cures available or methods to ease the suffering. In such situations, empathic healthcare can predispose patients to shifts in the perceptions of their body status, cause decreased reactivity to the underlying pathophysiology and relieve unnecessary suffering. ${ }^{9}$ In other words, patients who receive more empathic healthcare may have more hope and, consequently, relief. Interestingly, expectations or hopes can trigger bodily responses, particularly during childhood. ${ }^{10}$ Thus, placebos are necessary in RCTs and have some psychotherapeutic value.

Compared with well-targeted very efficacious medications, placebos should give only modest results in RCTs. ${ }^{11}$ An issue in RCTs is that controlled patients are not fully informed of whether they are specifically receiving a placebo treatment, ${ }^{12}$ so trials with placebo as the control sometimes pose an ethical dilemma if the test drug is effective. However, a placebo itself can indicate whether the 
efficacy of a new medical intervention or treatment is sufficient to justify its use. Notably, in the 1950s, Beecher performed a meta-analysis by combining the placebo treatment data collected from 15 studies on different diseases and found that placebos led to an approximately $35 \%$ improvement in symptoms. ${ }^{13}$ In some RCTs, placebos without any known active principles are of more help in relieving some patients' suffering than are tested medical treatments. The phenomenon of improvement of the symptoms of a patient who has taken a placebo in a RCT is called placebo effect or placebo response. ${ }^{14}$ The placebo effect is so robust that it can influence psychosocial and physiological responses to the placebo and to subsequent active treatments in many settings, so it has attracted increasing attention of researchers.

There are several theories that attempt to explain the mechanism of the placebo effect: the expectancy theory, classical conditioning accounts, context effects ${ }^{15}$ and the meaning response. ${ }^{16}$ Recently, a proposed framework based on integrative framework theory by Colloca and Miller ${ }^{17}$ has been widely accepted. The integrative framework theory emphasises that cues of a different nature (ie, verbal, contextual, social) may be integrated to generate key treatment expectancies, which can influence the effects of active or placebo treatment. Thus, empirical findings for placebo can be integrated into a single conceptual model rather than other complex dual mechanisms.

In this article, on the basis of the history of placebo, we summarise the characteristics of placebo effects and the recent advancements reported from studies on placebo effects, as well as highlight placebome studies to identify various molecular biological components of placebo effects.

\section{CHARACTERISTICS OF PLACEBO EFFECTS}

Placebo effects are actually the body's responses to a general expectancy through absorbing some cues, including physical and psychological ones. ${ }^{18}$ However, some major factors identified to affect placebo effects are patients' reporting bias, regression to the mean and the physiological variation of illnesses in RCTs. Since a patient may tend to report improvement that has not actually occurred under some circumstances, it is easy to treat reporting bias as a true effect of placebo based on subjective outcomes. ${ }^{19}$ The regression to the mean is a statistical phenomenon in which a variable is extreme on its first measurement, but with increases in the number of measurements, the variable's value approaches the mean or average,${ }^{20}$ that is, the placebo effect is high on the first measurement but may be lower on the second and subsequent measurements. Since the pathological conditions of many diseases undergo natural changes, some patients may have spontaneous remission along with the natural waxing and waning of an illness without any treatment or intervention. Thus, familiarity with the characteristics of placebo effects will provide a better understanding of how they work.

Placebo effects usually have the following characteristics: (1) since placebos have no inherent therapeutic power, they rarely cure the illness but may provide relief of some patients' subjective symptoms, such as pain; (2) placebo effects widely vary in patients with different diseases and in patients with the same disease treated with different medicines; ${ }^{21}$ (3) there are also adverse consequences of placebo effects, that is, the so-called 'nocebo effect'. Up to $26 \%$ of patients randomly assigned to placebos in RCTs are estimated to discontinue the use of placebos because these patients have perceived adverse effects. ${ }^{9}$ Actually, the psychosocial factors that induce nocebo effects can also cause adverse medication effects. ${ }^{4}$ Placebo effects are beyond the reach of medical intervention or treatment, the patients' cognitive level on the treatment and/or the physician-patient relationship can enhance the effectiveness of medical treatment. An interesting study demonstrated that the patients who took the real drug rizatriptan but labelled as 'placebo' had no different outcomes from those taking placebos labelled with 'rizatriptan'. But when patients took the real drug ritzatriptan correctly labelled as 'rizatriptan', the effect of this drug increased by about $50 \% .{ }^{22}$ Another study also obtained similar results in which the effects of open versus hidden administration of morphine for postoperative pain, beta-blockers for cardiovascular function, subthalamic stimulation for Parkinson's disease and diazepam for anxiety were compared, ${ }^{23}$ and open treatment was found to induce significantly greater improvement than that of the hidden one.

In the view of Miller and Colloca, ${ }^{24}$ the placebo effect is a learnt response generated by expectancies via the central nervous system. When a patient has an active or placebo treatment, the different cues may cause the patient to remember the previously experienced sensations and thereby develop an expectancy. ${ }^{17}$ Different cues can converge into a single conceptual model to generate key expectancies, which is a more general state that relates to consciousness or subconsciousness according to the specific process involved. To understand both placebo and nocebo effects, although they have different psychobiological mechanisms, the general conceptual framework is considered to be the same as the expectancies determined by prior experience. ${ }^{25}$ Increasing evidence supports the ideas that the placebo is not limited to inert agents but many active treatments may also have the similar effects of placebo. It is necessary to consider the placebo effect when carrying out any medical treatments.

\section{PLACEBO EFFECT STUDIES}

In the past, behavioural instruments were used to study the mechanism of placebo effects. ${ }^{4}$ Overall, behavioural studies suggest an important role of learning in the placebo effect, including individual training and social learning. It has been found that the individual training 
duration and method (continuous or partial reinforcement) may influence the results of various placebo effects, the verbal and social cues may influence the results of training and all combined available factors present during the clinical treatment may determine the overall results of placebo effects. Thus, there has been limited success for this approach because these instruments cannot explain the complex placebo response states that shift based on a patient's beliefs, expectations and previous experiences.

With advances in neuroimaging, we have explored a number of neurobiological mechanisms of the placebo effect. $^{2}$ Through this technique, placebo effects have been shown to be biological responses to psychosocial cues associated with the medical treatments that rely on complex neurotransmitters involved in neurobiological mechanisms, such as cannabinoids and dopamine, and on some brain regions, such as amygdala, anterior insula and prefrontal cortex in placebo analgesia. Although objective neurobiological pathways have been revealed to correlate with placebo effects, no evidence supports that placebo effects can alter the pathophysiology of diseases. ${ }^{26}$ These substantial advancements in the placebo effect are essential for evaluating drug effects.

To facilitate pharmaceutical development, rigorously characterised placebo effects based on each patient may be of great value to patients and researchers. For drug development, an underlying goal of RCTs is to find a difference between active treatment and the placebo control. ${ }^{27}$ Knowing likely placebo responders could improve trial designs to detect such a difference, and modify treatment approaches by allowing for more efficient medication dosages. A pressing issue in treatment is to solve the conflict of the disclosure of drugs' adverse effects with the avoidance inducing nocebo effects. ${ }^{9}$ Addressing this issue depends on characterised placebo effects based on each participant without deception. A striking finding in RCTs is the effect of possible placebo pathway genes on the treatment with both the placebo and drug, which has demonstrated that some drugs have placebo-drug interactions as a result of shared molecular targets. Thus, precise knowledge of molecular biological components of placebo effects promises to lead to greater understanding of the underlying mechanisms, although the environmental factors that surround a patient make it an ongoing challenge.

It has been widely accepted that clinical outcomes are affected by the interplay of genetic factors and environmental factors. ${ }^{28-30}$ As is known, the placebo effect is a complex phenotype affected by a subject's beliefs, expectations and previous experiences. Additionally, placebo effects were reported to have been observed in $77.5 \%$ of subjects in naltrexone trials for the treatment of alcoholism and there are other types of placebo effects that have been observed in patients with certain symptoms of pain, headache and nausea. ${ }^{31}$ Since an individual's genetic make-up is considered to be a stable inner trait, genetic variation is an important factor that influences placebo effects. ${ }^{32}$ Genetic variations can lead to function abnormality of genes, RNA and protein networks and form an individual's genetic response characteristics. ${ }^{29} 33$ Thus, greater understanding of genetic impacts on the placebo effect may help distinguish active treatment effects with placebo effects in certain research designs and help obtain the precise knowledge of the molecular biological components of specific placebo effects.

The recent availability of large-scale -omics data of genes, RNA and proteins (ie, genomics, epigenomics, transcriptomics, proteomics, and so on) offers a potential new approach to identifying molecular targets for placebo effects. The recently emerging concept of a placebome was proposed through collecting -omics data, such as genomic data, to unpack influences of genome-derived molecules on placebo effects. ${ }^{34}$ Since a better understanding of inner genetic influences of placebo effect is critical for evaluating and maximising the efficacy of medical treatment, knowledge of the placebome is of potential benefit to develop novel strategies for clinical trial designs, reduce trial cost and improve the understanding of the mechanisms underlying the placebo effect. Thus, placebome studies are justified for these reasons.

Despite the promise of placebome to discover and develop more effective personalised medicine, it is probably worth noting that, while still in a relatively nascent phase, '-omics' studies in psychiatry have yielded little tangible benefit to date regarding predictive therapeutic benefits (other than identifying individuals at risk for side effects due to variations in drug metabolism). It is also true that no genome-wide association studies (GWAS) related to placebo effects have yet been conducted. ${ }^{35}$ Thus, the search for genomic targets associated with placebo effects is in its infancy. In fact, many placebo-controlled RCTs have used genomics data, but all lacked a no treatment control (NTC), which is usually used to distinguish genuine placebo effects from regression to the mean and natural changes in an illness. Furthermore, in RCTs, addressing more ethical issues often takes precedence over use of an NTC since use of a placebo is thought to be treatment, but placebo employment without the patients' knowledge may violate their rights of informed consent and cause patients to distrust their doctors. ${ }^{14}$ In a recent placebome study without NTC, placebo effects varied between $25 \%$ and $75 \%$ in psychopharmacology, and because of the variable placebo effects, about $50 \%$ of antipsychotic clinical trials are not found to support the superiority of tested drugs over placebo, although the identified placebome module may be significantly similar to the depression and anxiety modules in the human interactome. ${ }^{21}$ Thus, inclusion of an NTC in studies to investigate the placebome is required in future studies.

From the viewpoint of Colloca and Miller, various responses can be integrated to generate key treatment expectancies; that is, different information into a single conceptual framework. Furthermore, whether the expectancies inducing the placebo effect require consciousness may depend on the specific process. In addition, the 
known cues that trigger placebo effects may be similar but not identical to previously experienced cues. Thus, there may be multiple mechanisms for placebo effects and there exists a key mechanism that has not been identified yet. In placebo analgesic studies, the learning mechanisms have been identified to affect neural and cognitive aspects of the placebo effects. ${ }^{17}$ In nocebo hyperalgesic studies, two regions, the rostral anterior cingulate cortex (rACC) and the periaqueductal grey (PAG), have been found to facilitate expectation-induced pain. Neuroimaging has shown that the neural interactions between the prefrontal areas, brainstem and spinal cord can mediate the nocebo effect. Through modulation of connecting within the rACC-PAG spinal axis, cognition interacts with the pain pathway to modulate pain and nociceptive processing at the spinal level. Further investigations of the molecular biological components that have yielded the behavioural and neuroimaging data are needed to elucidate the mechanisms of placebo or nocebo effects in greater depth. ${ }^{36}$

The placebome may consist of multiple intersecting pathways, and there may be genetic overlap between placebo, disease and treatment; specifically, the genes possibly involved in the placebo pathway exert effects in the drug pathway or disease pathogenic pathway. Based on previous studies of disorder treatments, the genes of placebo effects may have wide effects on dopamine, opioid, endocannabinoid and serotonin signalling pathways. ${ }^{34} 3738$ These four signalling pathways have been identified to affect neural and cognitive aspects of the placebo effect and are viewed as important processes in the subjective experience of symptom relief related to the placebo effect. ${ }^{34}$ In the next section, we summarise the molecular biological components involved in these signalling pathways.

\section{MOLECULAR BIOLOGICAL COMPONENTS OF PLACEBO EFFECTS}

The importance of identifying molecular biological components of the placebo effects is not limited to excluding the most likely placebo responders in RCTs to maximise the efficacy of medical treatments. Purposefully inhibiting the placebo effects by a drug in advance could minimise the placebo effect and interfere with evaluation of the effect of medical treatments in an objective manner. Through integrating the behavioural, neurobiological and genetic findings on the placebo effect, the dopamine, opioid, endocannabinoid and serotonin signalling pathways are used as the primary means for identifying molecular biological components through analysis of the genetic variants. ${ }^{16}$ However, it is worth noting that besides these four systems, various psychological and biological factors across different psychiatric diseases might also mediate placebo effects, but these have not been studied yet. Along with advances in knowledge about the neurotransmitters and neural pathways, increasingly specific candidate genes influencing the placebo effect have attracted greater attention. In particular, in the past years, high-throughput analysis technologies have produced a large number of gene and protein-protein interaction data that have stimulated studies of systems biology. These -omics data provide unprecedented opportunities to investigate the molecular targets of placebo effects at the systems level by conducting placebome studies. As mentioned earlier, certain genes or gene products may mediate placebo effects in individual patients together, and there is potential molecular overlap among placebo and medical treatment effects and the disease, which highlights the complexity of placebome studies and the importance of identifying the molecular biological components of placebo effects.

Table 1 summarises the genes with possible involvement in placebo effects, although multifaceted and complicated nature of placebo effects should be considered when reviewing the table. It is important to be aware that it would be unrealistic to expect several genetic variants alone to influence a majority of placebo effects because there is a lot of evidence supporting multiple mechanisms. ${ }^{39}$ This idea is also supported by a number of GWAS, which have demonstrated that almost all common variants affect complex traits with very small effect sizes. ${ }^{40}$ The genetic association studies require a certain number of samples to balance type I errors and power. Assessing multiple variants in one experiment may increase type I errors without any controls for multiple comparisons. Additionally, a significantly larger sample size is required to increase the power that may be reduced by controlling for multiple comparisons. Thus, the previous genetic studies of placebo effects with a relatively small number of participants may have underestimated and overestimated the role of some variants. To balance power and type I errors, a study design using small twin or sibling samples to investigate the genetic contribution to placebo effects is plausible to increase power and reduce noise. This approach could obtain the highly similar genetic background, and therefore clearly identify the possible genes involved in placebo effects. An increasing number of studies have demonstrated that the neurotransmitter and neurological pathways can mediate placebo effects, and have provided candidate genes for further studies. First, a placebo was found to induce the pain suppression system of the body, which can be blocked by an opioid receptor antagonist. ${ }^{41}$ 42 This finding demonstrated that the opioid signalling pathways may be involved in a placebo analgesic effect. Furthermore, activation of some brain regions induced by expectation of analgesia is related to endogenous opioid transmission and analgesia. Further physiological experiments have demonstrated that the endocannabinoid signalling pathway is also implicated in the placebo analgesia. ${ }^{43}$ Based on the finding of analgesic effects of opioid receptor signalling, expectancy of reward is postulated as a key general contributor to the placebo effect. In a pain model, anticipation of the placebo effect stimulated the activation of opioid and dopamine receptor in brain, and higher placebo effects have been found to correspond to higher levels of dopamine receptor activation. Additionally, both 


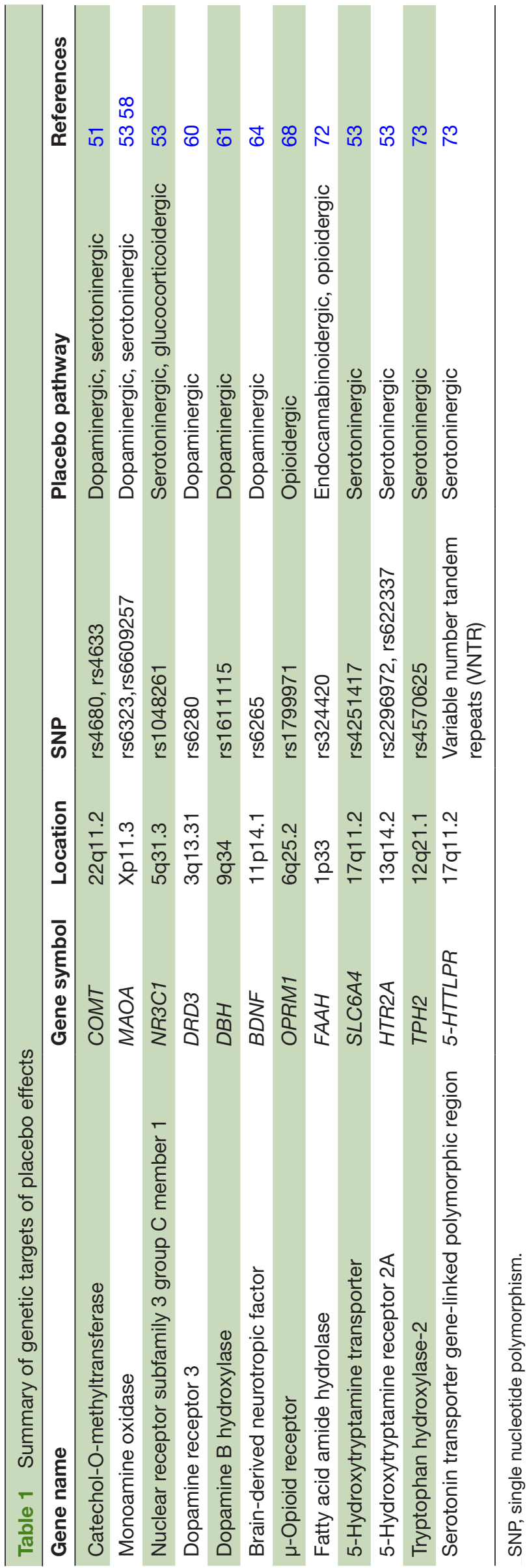

dopaminergic and opioid signallings were found to be reduced in individuals with negative placebo effects. ${ }^{42}$ Furthermore, the serotonin signalling pathway has major roles in depression, ${ }^{44}$ which causes a much higher rate of placebo effects in RCTs. Placebo treatment in depression RCTs has been shown to cause changes in brain function. Thus, mutation of genes involved in the metabolism and signalling of the neurotransmitters in the signalling pathways described below may influence the results of placebo effects and help us understand the effects, although the evidence supporting implication of the endocannabinoid and serotonin pathways in the placebo effect is more limited.

\section{Dopamine signalling pathways}

Dopamine signalling pathways, which are dopaminergic projections, are the sets of projection neurons that synthesise and communicate the neurotransmitter dopamine. Individual neurons in these pathways are called dopamine neurons, which have axons along the entire length of the pathway. Dopaminergic pathways regulate many functions, such as motivation, learning, reward and neuroendocrine control, and are involved in multiple psychiatric and neurological disorders. Dopamine neurotransmission pathways are also related to pain syndromes, including headache and postoperative pain. ${ }^{16}$ Genetic factors modifying dopaminergic signalling can also regulate the brain reward circuit. ${ }^{45}$ Since the dopamine-mediated reward centres also are part of the physiology involved in the placebo effects, the genes involved in dopamine metabolism and the signalling pathway may be prime candidate molecular targets for investigation of placebo effects.

The catechol-O-methyltransferase (COMT) is an enzyme that catalyses the transfer of a methyl group from S-adenosylmethionine to endogenous catecholamines, such as the neurotransmitters dopamine. ${ }^{46}$ This O-methylation causes degradation of catecholamine transmitters. Because COMT can influence dopamine brain levels, it is considered to affect the extent of the placebo effect. A single nucleotide polymorphism (SNP) $r s 4680(\mathrm{G}>\mathrm{A})$ in COMT encoding a valine ( $\mathrm{G}$ allele) to methionine (A allele) amino acid (AA) substitution at codon 158 has been found to reduce the enzymatic activity of COMT by threefold to fourfold. ${ }^{47}$ Particularly, the homozygous form of the A allele has repeatedly been found to be related to reduced dopamine level in the prefrontal cortex. ${ }^{348}$ Rs4680 is a common polymorphism, and according to the 1000 Genomes Project Phase 3, the prevalence of the minor allele A is 0.28 in East Asians and 0.5 in Europeans. ${ }^{49}$ Because reduced dopaminergic activity is related to high pain sensitivity, rs4680 has been implicated in influencing test outcomes in numerous trials ranging from psychiatric disorders to cardiovascular diseases and irritable bowel syndrome (IBS). ${ }^{50}$

Until now, only one genetic association study has included a NTC group, and that study examined the association of variants in COMT with placebo effects. ${ }^{51}$ This 
study was based on a previous RCT with three groups, that is, an NTC group, a placebo acupuncture group and a placebo acupuncture+warmer caring group, to evaluate placebo acupuncture treatment of IBS. ${ }^{52}$ The results of that RCT showed that the best placebo treatment induced the strongest symptom relief since the RCT design potentially ruled out some factors affecting the responses to placebo treatment. Further, the genetic analysis results suggested that subjects in the homozygote of the rs4680 AA genotype with low enzyme activity resulting in high levels of dopamine in the body had the greatest placebo responses, and the $\mathrm{G}$ allele homozygous patients with high enzyme activity had the lowest placebo response. The heterozygotes of the GA genotype had an intermediate response. Moreover, another SNP rs4633, in the linkage with rs4680, had been found to give similar results.

To the best of our knowledge, to date the largest study of genetic variations in RCT patients with placebo and bupropion treatments for major depressive disorder examined a total of 532 variants in 34 candidate genes. ${ }^{53}$ Although there were no results for rs 4680 in that study, several other SNPs in COMT were found to be significantly associated with placebo effects. However, after the correction for multiple comparisons, these SNPs were not found to be associated with placebo effects. Interestingly, in a recent laboratory study, the G allele of rs 4680 related to the high enzyme activity of COMT was found to be significantly associated with a higher frequency of nocebo effects and complaint record. ${ }^{55}$ The finding suggests that when individuals with the GG genotype show absence of any significant improvements in symptoms, they may have more side effects, such as complaints and nocebo effects. However, this result must be verified in a wide population since the study primarily tested Caucasian women.

In addition to COMT, there are several other genetic target candidates of placebo effects in the dopamine pathway. The monoamine oxidase A (MAOA) gene encodes an enzyme bound to the outer membrane of mitochondria in most cell types and can catalyse the oxidative deamination of amines, such as dopamine, serotonin and norepinephrine, and thus takes part in reward pathways and affects serotonergic signalling pathways. ${ }^{56} \mathrm{~A}$ common SNP rs6323 (G>T) in MAOA has been found to reduce the enzymatic activity by $75 \%$ in individuals with the only allele of $\mathrm{T}^{57}$ The first association study of rs6323 in the gene $M A O A$ with placebo effects recruited patients with clinical depression from four placebo-controlled RCTs. ${ }^{58}$ Individuals containing the low-activity MAOA genotypes, which cause higher basal dopamine level, had higher placebo effects. However, in this study, rs4680 in the COMT gene was not found to be significantly associated with placebo effects, which may be because of a lack of statistical power, the subject difference or study design without a NTC group. In the above-mentioned largest genetic association study of placebo treatment, which sacrificed statistical power to detect variants with significant associations of placebo effects, the SNP rs6609257 within the gene $M A O A$ involved in dopamine basal tone as well as rs1048261 in the nuclear receptor subfamily 3 group C member 1 (NR3C1) gene was found to be significantly associated with placebo-induced improvement in depression. ${ }^{53}$ The NR3C1 protein is a glucocorticoid receptor usually staying in the cytoplasm. On ligand binding, NR3C1 can be transported into the nucleus and functions both as a transcription factor and regulator of other transcription factors to mainly regulate the transcription of glucocorticoid responsive genes. These findings support that the MAOA and NR3C1 genes should be considered as molecular targets of placebo effects.

The dopamine receptor 3 (DRD3) encodes the D3 subtype of the five dopamine receptors, which are mediated by $\mathrm{G}$ proteins primarily located in the olfactory tubercle, nucleus accumbens and islands of Calleja in the brain, and are involved in cognitive, emotional and endocrine functions. A common exonic rs6280 (C>T) in the gene $D R D 3$ can cause an AA substitution of glycine to serine at codon 9 (Gly9Ser). The mutant $D R D 3$ with serine has been found to have a lower affinity for dopamine. $^{59}$ A recent RCT of a novel drug (ABT-925) for treating schizophrenia examined the effects of variants in the DRD 3. ${ }^{60}$ Patients with homozygous T allele of rs6280 in $D R D 3$ gene were found to have significantly better outcomes in the group of placebo treatment than in the group of ABT-95 treatment with increasing doses. That study demonstrated that DRD3 should be a molecular target of the placebo effects, and supports that subjects homozygous for rs 4680 A allele in the COMT gene have a greater placebo response.

The dopamine beta-hydroxylase $(D B H)$ is an oxidoreductase belonging to the copper type II, ascorbate-dependent mono-oxygenase family. DBH is mainly expressed in neuroscretory vesicles and chromaffin granules of the adrenal medulla, and converts dopamine to norepinephrine. This enzyme has two forms, that is, soluble and membrane-bound forms. DBH has been reported to be associated with deficits in autonomic and cardiovascular function and psychiatric diseases. In the alcohol dependence studies, individuals with homozygous $\mathrm{C}$ allele of the rs 1611115 in the $D B H$ gene appeared to have better symptom improvement on the group of placebo treatment than on the group of naltrexone treatment. ${ }^{61}$ $D B H$ was also examined in the largest genetic association study of the placebo and bupropion treatment for the major depressive disorder mentioned above. ${ }^{53}$ The SNP rs2873804 in the $D B H$ gene was found to be significantly associated with placebo effects after the correction for multiple comparisons, which reinforces $D B H$ as a potential molecular target for a placebo effect.

The brain-derived neurotrophic factor (BDNF) is a member of the nerve growth factor family of proteins. BDNF may promote neuronal survival and differentiation in the peripheral and central nervous systems, and participate in the modulation of axonal and dendritic growth 
and morphology. Being a major regulator of synaptic transmission and plasticity in the adult brain, BDNF has versatile roles in a range of adaptive neuronal responses, such as long-term depression, long-term potentiation, as well as homeostatic regulation of intrinsic neuronal excitability. Furthermore, BDNF has been found to regulate the stress response, modulate the pathogenesis of mood disorders and maintain turnover of dopamine. ${ }^{62}$ The functions of BDNF in neuroadaptive change and response to reward stimuli have attracted researchers' interest in identifying plausible candidates involved in placebo effects. ${ }^{63}$ The SNP rs6265 (C>T) in the BDNF gene causes a valine to methionine substitution at codon 66 (Val66Met), which results in inefficient BDNF trafficking to secretory granules. ${ }^{46}$ Furthermore, the $\mathrm{C}$ allele of rs6265 has been found to be associated with greater placebo-induced activation of dopamine receptors D2 and D3 than that of $\mathrm{T}$ allele carriers; however, this gene was not found to be significantly associated with placebo analgesia despite that the SNP rs6265 has been hypothesised to reduce activity-dependent BDNF release. ${ }^{64}$

Collectively, the results of association studies of placebo effects with dopamine-related genetic variants support the genes involved in dopamine pathway as molecular targets of placebo effects. More research with larger samples in studies that include NTCs would help provide definitive results.

\section{Opioid signalling pathways}

This system consists of multiple molecular signals generated by an opioid receptor binding to its physiological ligands. Opioids, broadly used as pain killers, are chemical substances extracted from opium (morphine, codeine, and so on), which possess strong analgesic and sedative effects. Opioid receptors are G-protein coupled receptors that are widely distributed throughout the human body and crucially involved in pain signalling in the central and peripheral nervous systems, and immunological response, and so on. There are four classes of receptors: $\mathrm{mu}$ ( $\mu$-opioid receptor, OPRM), kappa, delta and nociceptin. In studies of placebo analgesic effects, both the endogenous opioid and dopaminergic signalling pathways have been found to be activated. Especially, in antinociceptive responses to placebo, opioid receptor signalling has been found to be entangled with the dopamine signalling pathways. ${ }^{65}$

Furthermore, OPRM1 has been found to affect the clinical outcomes of pain treatment in studies of placebo analgesia. The mutation of the A allele to $\mathrm{G}$ allele of rs1799971 in OPRM1 can cause an asparagine to aspartic acid alteration at codon 40 receptor, which may reduce the expression and function of OPRM1. ${ }^{667}$ The functional aspartic acid (G) allele of rs1799971 carriers has been found to have lower placebo-involved activation of dopamine neurotransmission unlike the asparagine (A) allele homozygotes, which suggests that genetic variation in OPRM1 could also contribute to variability of the placebo effects. ${ }^{68}$ In that same study, using positron emission tomography (PET) technology and radio tracers to label $\mu$-opioid and dopamine receptors, compared with $\mathrm{G}$ allele carriers, the allele AA homozygotes of the functional rs1799971 showed an increase in the baseline level of OPRM in brain areas in response to pain and mood. Following a placebo treatment, $G$ allele carriers were correlated with higher Neuroticism Extraversion Openness (NEO) personality scores and showed lower levels of mood, OPRM and dopamine receptor activation in the thalamus, nucleus accumbens and anterior insula. These findings implicate OPRM1 in the placebo-involved modulation and individual differences in neurotransmission. However, association studies of genetic variation in OPRM1 with the addictive effects of opioid drugs and psychostimulants (eg, amphetamine) have obtained conflicting outcomes. ${ }^{6169}$ That conflict may provide some support for the need of an NTC to determine the genetic variation caused by differences in placebo effects.

\section{Endocannabinoid and serotonin signalling pathways}

Endocannabinoids (endogenous cannabinoids) are endogenous lipid-based retrograde neurotransmitters and include at least five derivatives of arachidonic acid, such as: arachydonoyl ethanolamide and 2-arachydonoil glycerol. They are released from postsynaptic neurons and bind to cannabinoid receptors, specifically cannabinoid type 1 (CB1) and CB2. The endocannabinoid signalling pathways are involved in regulating a variety of physiological and cognitive processes (such as appetite, pain sensation, mood and exercise-induced euphoria) and in mediating the pharmacological effects of cannabis. CB1 receptors are predominantly expressed in the peripheral and central nervous systems, and are mainly activated by the endocannabinoids, anandamide, as well as its mimetic phytocannabinoid, tetrahydrocannabinol. ${ }^{70}$ Antagonist-based placebo analgesia studies have supported that endocannabinoid is involved in placebo analgesia. ${ }^{38}$

The fatty acid amide hydrolase (FAAH) located on chromosome 1p33 is an integral membrane protein, which is responsible for the hydrolysis of some bioactive fatty acid amides, such as the neuromodulatory compounds anandamide and oleamide. Serving as the major degradative enzyme of endocannabinoids, FAAH may play roles in endocannabinoid responses to pain and placebo analgesia. The genetic variation in $F A A H$ has been examined in a small study with the same subjects as mentioned above. ${ }^{68}$ The SNP rs324420 $(\mathrm{C}>\mathrm{A})$ within the $F A A H$ gene encodes a missense substitution of proline to threonine at codon 129 (Pro129Thr). It has been reported that in response to pain subjects with homozygotes for the $\mathrm{C}$ allele of rs324420 had increased endocannabinoid levels in the brain, and a greater placebo analgesic response, and improved mood. ${ }^{72}$ These findings support that the endocannabinoid pathway genes are potential candidate molecular targets of placebo effects that are worth exploring further.

Serotonin is an important hormone and neurotransmitter with many roles. The serotonin signalling pathways 
are a set of projection neurons in the brain, including rostral and caudal groups, which synthesise and communicate the monoamine neurotransmitter serotonin. Individual neurons in these pathways are called as serotonergic neurons. Since the serotonergic neurons innervate wide places, these pathways regulate mood, appetite and sleep and are relevant to many psychiatric and neurological disorders.

Since the high incidence of placebo effects in RCTs of placebo treatments for mood disease, the serotonin pathway could plausibly be examined for possible placebo effect-related genes. ${ }^{34}$ In the above-mentioned study, in which the association of 34 possible genes was examined with placebo effects, several genes involved in the serotonergic pathway were significantly related to placebo remission, including solute carrier family 6 member 4 (SLC6A4) rs4251417 and 5-hydroxytryptamine receptor 2A (HTR2A) rs2296972 and rs622337. ${ }^{53}$ SLC6A4 is an integral membrane protein that can terminate the action of serotonin and recycle it in a sodium-dependent manner through transporting the serotonin from synaptic spaces into presynaptic neurons. HTR2A is a guanine nucleotide-binding protein (G-proteins) coupled receptor for 5-hydroxytryptamine (serotonin) that plays a role in the regulation of behaviours, such as responses to anxiogenic situations and psychoactive substances, and in intestinal smooth muscle contraction and arterial vasoconstriction. Receptor ligand binding causes a conformational change in HTR2A that triggers intracellular signalling via G-proteins and modulates the activity of downstream effectors.

Moreover, serotonin-involved placebo effect genes have been examined in a social anxiety disorder (SAD) RCT. In the genetic PET study of SAD, a reduction in stress-related amygdala activity was accompanied by a reduction in anxiety symptoms under the treatment of placebo. ${ }^{73}$ Only subjects homozygous for rs4570625 $\mathrm{T}$ allele within the tryptophan hydroxylase-2 (TPH2) gene promoter and the long allele of the serotonin transporter-linked polymorphic region (5-HTTLPR) have such a reduction. TPH2 is a member of the pterin-dependent aromatic acid hydroxylase family, which catalyses the first and rate-limiting step in the biosynthesis of serotonin. 5-HTTLPR is a polymorphic region located within the $5^{\prime}$ regulatory region of the SLC6A4 gene including the promoter, which is composed of 16 tandemly repeated units in a long (L) allele, and deletion of repeat units $6-8$ in a short (S) allele, where each repeat unit is 20-23 bps in length. The 5-HTTLPR region may have both positive and negative-acting cis-regulation on the expression of SLC6A4. Moreover, the SNPs of rs25531 and rs25532 have also been identified within 5-HTTLPR.

Although most genetic association studies of placebo effects have several limitations, such as small size and no NCT, the genes in table 1 are potential molecular biological components of placebo effects in different disorders. However, more data are required to precisely define the roles of dopamine, opioid, endocannabinoid and serotonin based on the genetic background of placebo effects.
Recently, a placebome analysis based on the known seed genes influencing placebo effects tried to identify a subnetwork of interacting proteins involved in placebo effects. ${ }^{21}$ In this analysis, a placebome module constructed with an interactome of genes or proteins was identified to be significantly close to the vascular disease modules. Moreover, diseases with molecular network modules very close to the placebome module might be candidates for placebo as potential 'drugs'. For drugs targeting placebome module molecules, placebo effects may influence the drug test outcome. Furthermore, genetic variants in placebome module genes that modify the placebo effects, such as COMT, may provide a genetic target or biomarker of place effects. In this study, an indirect cohort was used to validate these findings by examining whether the placebome module had more genes with SNPs significantly related to a placebo effect than a random situation. Although a strict validation is required, this study suggests that the interaction between diseases/ drug targets and the placebome module would tend to be stable. Given the complex interplay of expectation, behaviour and disease, a potentially complex network in the context of genes and environmental factors may determine placebo effects. Considering the complexity and lack of sufficient data, additional placebome studies are required.

The placebo effect is complex, and its physiology is incompletely understood. In previous studies, multiple intersecting pathways were found to be integrated into four signalling systems. In the viewpoint of Colloca and Miller, the core factors identified so far require further investigations to understand how they interact in complex biological networks.

\section{CONCLUSION AND PERSPECTIVE}

Placebo has a long history, but research on the genetics related to the placebo effect is in the early stage. In particular, research on placebome is in its infancy. A number of behavioural and neurobiological studies have explored the mechanism of placebo effects, including how they are stimulated. But the underlying mechanisms of placebo effect have not been fully understood. Although some genetic studies have been rigorously conducted, very few have explored the multiple possible genes in one time, like placebome studies. Then a primary limitation of this review is that it relies on a limited number of studies, so caution is required when considering these very preliminary conclusions. Although the molecules in the dopamine, opioid, serotonin and endocannabinoid systems might be targets for involvement in placebo effects on the basis of the combination of behavioural, neurobiological and genetic findings, placebome studies are required to identify genetic targets in the scope of the entire genome. Although bioinformatics analysis has identified the molecular placebome module, placebome studies with NTCs are also required to validate the related findings. 
Contributors LC conceived and wrote the whole manuscript. LH proofread the manuscript.

Funding This work was supported by the Ministry of Science and Technology Precision Medicine Project (No 2017YFC0909200 and No 2017YFC1001300) and the Natural Science Foundation of Shanghai (No 19ZR1427700).

Competing interests None declared.

Patient consent for publication Not required.

Provenance and peer review Commissioned; externally peer reviewed.

Data availability statement Data is available from the authors on request

Open access This is an open access article distributed in accordance with the Creative Commons Attribution Non Commercial (CC BY-NC 4.0) license, which permits others to distribute, remix, adapt, build upon this work non-commercially, and license their derivative works on different terms, provided the original work is properly cited, appropriate credit is given, any changes made indicated, and the use is non-commercial. See: http://creativecommons.org/licenses/by-nc/4.0/.

\section{REFERENCES}

1 Lasagna L. The placebo effect. J Allergy Clin Immunol 1986;78:161-5

2 Finniss DG. Placebo effects: historical and modern evaluation. Int Rev Neurobiol 2018;139:1-27.

3 Kerr CE, Milne I, Kaptchuk TJ. William Cullen and a missing Mind-body link in the early history of placebos. J $R$ Soc Med 2008;101:89-92.

4 Jütte R. The early history of the placebo. Complement Ther Med 2013;21:94-7.

5 De Craen AJM, Kaptchuk TJ, Tijssen JGP, et al. Placebos and placebo effects in medicine: historical overview. J $R$ Soc Med 1999:92:511-5.

6 Fregni F, Imamura M, Chien HF, et al. Challenges and recommendations for placebo controls in randomized trials in physical and rehabilitation medicine: a report of the International placebo symposium Working group. Am J Phys Med Rehabil 2010;89:160-72.

7 Keränen T, Halkoaho A, Itkonen E, et al. Placebo-Controlled clinical trials: how trial documents justify the use of randomisation and placebo. BMC Med Ethics 2015;16:2.

8 Yang B, Wei J, Ju P, et al. Effects of regulating intestinal microbiota on anxiety symptoms: a systematic review. General Psychiatry 2019;32:e100056.

9 Kaptchuk TJ, Miller FG. Placebo effects in medicine. New England Journal of Medicine 2015;373:8-9.

10 Linde K, Fässler M, Meissner K. Placebo interventions, placebo effects and clinical practice. Philosophical Transactions of the Royal Society B: Biological Sciences 2011;366:1905-12.

11 Zheng W, Cai D-B, Li H-Y, et al. Adjunctive Peony-Glycyrrhiza decoction for antipsychotic-induced hyperprolactinaemia: a meta-analysis of randomised controlled trials. Gen Psychiatr 2018;31:e100003.

12 Millum J, Grady C. The ethics of placebo-controlled trials: methodological justifications. Contemp Clin Trials 2013;36:510-4.

13 Beecher HK. The powerful placebo. J Am Med Assoc 1955;159:1602-6.

14 Finniss DG, Kaptchuk TJ, Miller F, et al. Biological, clinical, and ethical advances of placebo effects. Lancet 2010;375:686-95.

15 Blasi ZD, Harkness E, Ernst E, et al. Influence of context effects on health outcomes: a systematic review. Lancet 2001;357:757-62.

16 Colagiuri B, Schenk LA, Kessler MD, et al. The placebo effect: from concepts to genes. Neuroscience 2015;307:171-90.

17 Colloca L, Miller FG. How placebo responses are formed: a learning perspective. Philosophical Transactions of the Royal Society B: Biological Sciences 2011;366:1859-69.

18 Rutherford BR, Mori S, Sneed JR, et al. Contribution of spontaneous improvement to placebo response in depression: a meta-analytic review. J Psychiatr Res 2012;46:697-702.

19 Hróbjartsson A, Gøtzsche PC. Is the placebo powerless? an analysis of clinical trials comparing placebo with no treatment. $N$ Engl J Med 2001;344:1594-602.

20 Bland JM, Altman DG. Regression towards the mean. Bmj 1994;308.

21 Wang R-S, Hall KT, Giulianini F, et al. Network analysis of the genomic basis of the placebo effect. JCI Insight 2017;2.

22 Láinez MJA. Rizatriptan in the treatment of migraine. Neuropsychiatr Dis Treat 2006;2:247-59.
23 Benedetti F, Carlino E, Pollo A. How placebos change the patient's brain. Neuropsychopharmacology 2011;36:339-54.

24 Miller FG, Colloca L. Semiotics and the placebo effect. Perspect Biol Med 2010;53:509-16.

25 Turi Z, Bjørkedal E, Gunkel L, et al. Evidence for cognitive placebo and nocebo effects in healthy individuals. Sci Rep 2018;8:17443.

26 Wager TD, Atlas LY. The neuroscience of placebo effects: connecting context, learning and health. Nat Rev Neurosci 2015;16:403-18.

27 Misra S. Randomized double blind placebo control studies, the "Gold Standard" in intervention based studies. Indian J Sex Transm Dis AIDS 2012;33:131-4.

28 Cai L, Deng S-L, Liang L, et al. Identification of genetic associations of SP110/MYBBP1A/RELA with pulmonary tuberculosis in the Chinese Han population. Hum Genet 2013;132:265-73.

29 Cai L, Huang T, Su J, et al. Implications of newly identified brain eQTL genes and their interactors in schizophrenia. Mol Ther Nucleic Acids 2018;12:433-42.

30 Cai L, Yuan W, Zhang Z, et al. In-Depth comparison of somatic point mutation callers based on different tumor next-generation sequencing depth data. Sci Rep 2016;6:36540.

31 Litten RZ, Castle I-JP, Falk D, et al. The placebo effect in clinical trials for alcohol dependence: an exploratory analysis of 51 naltrexone and acamprosate studies. Alcohol Clin Exp Res 2013;37:2128-37.

32 Maclin JMA, Wang T, Xiao S. Biomarkers for the diagnosis of Alzheimer's disease, dementia Lewy body, frontotemporal dementia and vascular dementia. General Psychiatry 2019;32:e100054.

33 Huang T, Liu C-L, Li L-L, et al. A new method for identifying causal genes of schizophrenia and anti-tuberculosis drug-induced hepatotoxicity. Sci Rep 2016;6:32571

34 Hall KT, Loscalzo J, Kaptchuk TJ. Genetics and the placebo effect: the placebome. Trends Mol Med 2015;21:285-94.

35 Cai L, Zheng LA, He L. The forty years of medical genetics in China. $J$ Genet Genomics 2018;45:569-82.

36 Tinnermann A, Geuter S, Sprenger C, et al. Interactions between brain and spinal cord mediate value effects in nocebo hyperalgesia. Science 2017;358:105-8.

37 Zubieta J-K, Bueller JA, Jackson LR, et al. Placebo effects mediated by endogenous opioid activity on mu-opioid receptors. J Neurosci 2005;25:7754-62.

38 Benedetti F, Amanzio M, Rosato R, et al. Nonopioid placebo analgesia is mediated by CB1 cannabinoid receptors. Nat Med 2011;17:1228-30.

39 Benedetti F, Pollo A, Lopiano L, et al. Conscious expectation and unconscious conditioning in analgesic, motor, and hormonal placebo/nocebo responses. The Journal of Neuroscience 2003;23:4315-23.

40 Gibson G. Rare and common variants: twenty arguments. Nat Rev Genet 2012;13:135-45.

41 Eippert F, Bingel U, Schoell ED, et al. Activation of the opioidergic descending pain control system underlies placebo analgesia. Neuron 2009;63:533-43.

42 Sobczak M, Sałaga M, Storr MA, et al. Physiology, signaling, and pharmacology of opioid receptors and their ligands in the gastrointestinal tract: current concepts and future perspectives. $J$ Gastroenterol 2014;49:24-45

43 Zou S, Kumar U. Cannabinoid receptors and the endocannabinoid system: signaling and function in the central nervous system. Int $J$ Mol Sci 2018;19.

44 Kraus C, Castrén E, Kasper S, et al. Serotonin and neuroplasticity links between molecular, functional and structural pathophysiology in depression. Neuroscience \& Biobehavioral Reviews 2017;77:317-26.

45 Diaz J, Pilon C, Le Foll B, et al. Dopamine D3 receptors expressed by all mesencephalic dopamine neurons. J Neurosci 2000;20:8677-84.

46 Vaccarino V, Brennan M-L, Miller AH, et al. Association of major depressive disorder with serum myeloperoxidase and other markers of inflammation: a twin study. Biol Psychiatry 2008;64:476-83.

47 Lotta T, Vidgren J, Tilgmann C, et al. Kinetics of human soluble and membrane-bound catechol O-methyltransferase: a revised mechanism and description of the thermolabile variant of the enzyme. Biochemistry 1995;34:4202-10.

48 Meyer-Lindenberg A, Kohn PD, Kolachana B, et al. Midbrain dopamine and prefrontal function in humans: interaction and modulation by COMT genotype. Nat Neurosci 2005;8:594-6.

49 Auton A, Brooks LD, Durbin RM, et al. A global reference for human genetic variation. Nature 2015;526:68-74.

50 Tammimaki A, Mannisto PT. Catechol-O-Methyltransferase gene polymorphism and chronic human pain: a systematic review and meta-analysis. Pharmacogenetics and genomics 2012;22:673-91.

51 Hall KT, Lembo AJ, Kirsch I, et al. Catechol-O-Methyltransferase Val158Met polymorphism predicts placebo effect in irritable bowel syndrome. PLoS One 2012;7:e48135. 
52 Kaptchuk TJ, Kelley JM, Conboy LA, et al. Components of placebo effect: randomised controlled trial in patients with irritable bowel syndrome. BMJ 2008;336:999-1003.

53 Tiwari AK, Zai CC, Sajeev G, et al. Analysis of 34 candidate genes in bupropion and placebo remission. Int $J$ Neuropsychopharmacol 2013;16:771-81.

54 Wendt L, Albring A, Benson S, et al. Catechol-O-Methyltransferase Val158Met polymorphism is associated with somatosensory amplification and nocebo responses. PLoS One 2014;9:e107665.

55 Hall KT, Tolkin BR, Chinn GM, et al. Conscientiousness is modified by genetic variation in catechol- $O$-methyltransferase to reduce symptom complaints in IBS patients. Brain Behav 2015;5:39-44.

56 Mickey BJ, Ducci F, Hodgkinson CA, et al. Monoamine oxidase a genotype predicts human serotonin $1 A$ receptor availability in vivo. $J$ Neurosci 2008;28:11354-9.

57 Hotamisligil GS, Breakefield XO. Human monoamine oxidase a gene determines levels of enzyme activity. Am J Hum Genet 1991;49:383-92.

58 Leuchter AF, McCracken JT, Hunter AM, et al. Monoamine oxidase A and catechol-O-methyltransferase functional polymorphisms and the placebo response in major depressive disorder. J Clin Psychopharmacol 2009;29:372-7.

59 Lundstrom K, Turpin MP, Large C, et al. Mapping of dopamine D3 receptor binding site by pharmacological characterization of mutants expressed in $\mathrm{CHO}$ cells with the Semliki Forest virus system. $J$ Recept Signal Transduct Res 1998;18:133-50.

60 Bhathena A, Wang Y, Kraft JB, et al. Association of dopamine-related genetic loci to dopamine D3 receptor antagonist ABT-925 clinical response. Trans/ Psychiatry 2013;3:e245.

61 Arias AJ, Gelernter J, Gueorguieva R, et al. Pharmacogenetics of naltrexone and disulfiram in alcohol dependent, dually diagnosed veterans. Am J Addict 2014;23:288-93.

62 Goggi J, Pullar IA, Carney SL, et al. Modulation of neurotransmitter release induced by brain-derived neurotrophic factor in rat brain striatal slices in vitro. Brain Res 2002;941:34-42.
63 Krishnan V, Han M-H, Graham DL, et al. Molecular adaptations underlying susceptibility and resistance to social defeat in brain reward regions. Cell 2007;131:391-404.

64 Pecina M, Martinez-Jauand M, Love T, et al. Valence-specific effects of BDNF Val66Met polymorphism on dopaminergic stress and reward processing in humans. J Neurosci 2014;34:5874-81.

65 Scott DJ, Stohler CS, Egnatuk CM, et al. Placebo and nocebo effects are defined by opposite opioid and dopaminergic responses. Arch Gen Psychiatry 2008;65:220-31.

66 Zhang Y, Wang D, Johnson AD, et al. Allelic expression imbalance of human mu opioid receptor (OPRM1) caused by variant A118G. J Biol Chem 2005;280:32618-24.

67 Kroslak T, LaForge KS, Gianotti RJ, et al. The single nucleotide polymorphism A118G alters functional properties of the human mu opioid receptor. J Neurochem 2007;103:77-87.

68 Peciña M, Love T, Stohler CS, et al. Effects of the mu opioid receptor polymorphism (OPRM1 A118G) on pain regulation, placebo effects and associated personality trait measures. Neuropsychopharmacology 2015;40:957-65.

69 Chen AC, Morgenstern J, Davis CM, et al. Variation in mu-opioid receptor gene (OPRM1) as a Moderator of naltrexone treatment to reduce heavy drinking in a high functioning cohort. J Alcohol Drug Depend 2013;1.

70 Hohmann AG. Spinal and peripheral mechanisms of cannabinoid antinociception: behavioral, neurophysiological and neuroanatomical perspectives. Chem Phys Lipids 2002;121:173-90.

71 Colloca L, Sigaudo M, Benedetti F. The role of learning in nocebo and placebo effects. Pain 2008;136:211-8.

72 Peciña M, Martínez-Jauand M, Hodgkinson C, et al. FAAH selectively influences placebo effects. Mol Psychiatry 2014;19:385-91.

73 Furmark T, Appel L, Henningsson S, et al. A link between serotoninrelated gene polymorphisms, amygdala activity, and placeboinduced relief from social anxiety. J Neurosci 2008;28:13066-74.

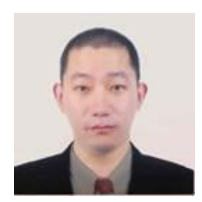

Lei Cai is an associated professor of Shanghai Jiaotong University, information commissioner offiaotong University library, a youth editor for the Journal "Chinese medical ethics". He obtainedthe Ph.D degree from Fudan University, China and received Science trainings from Harvard Schoolof public health, US. His research interests are focused on study the molecular basis of mind-bodyinteraction and Big data analysis. Till now, over 40 papers and four chapters for two books havebeen published, two invention patents have been authorized. He has been PI of several projects orresearch tasks, such as National Natural Science Foundation of China/ Shanghai, the Ministry ofEducation (MOE) Scientific Research Foundation, and the key research and development program ofthe Ministry of Science and Technology. 